\title{
What Happens to the Articular Surface After Curettage for Epiphyseal Chondroblastoma? A Report on Functional Results, Arthritis, and Arthroplasty
}

\author{
Germán L. Farfalli MD, Pablo A. I. Slullitel MD, D. Luis Muscolo MD, \\ Miguel A. Ayerza MD, Luis A. Aponte-Tinao MD
}

Published online: 1 February 2016

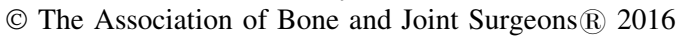

\begin{abstract}
Background Chondroblastoma is an uncommon, benign, but locally aggressive bone tumor that occurs in the apophyses or epiphyses of long bones, primarily in young patients. Although some are treated with large resections, aggressive curettage and bone grafting are more commonly performed to preserve the involved joint. Such intralesional resection may result in damage to the growth plate and articular cartilage, which can result in painful arthritis. Prior studies have focused primarily on oncologic outcomes rather than long-term joint status and functional outcomes.

Questions/purposes (1) What local complications can be expected after aggressive intralesional curettage of epiphyseal chondroblastoma? (2) What is the joint survival of a joint treated in this way for chondroblastoma? (3) What additional procedures are used in treating symptomatic joint osteoarthritis after treatment of the chondroblastoma? (4) What are the functional outcomes in this group of patients? Methods A retrospective study of our prospectively collected database between 1975 and 2013 was done. We
\end{abstract}

One of the authors certifies that he (LAA-T) or a member of his immediate family, has or may receive payments or benefits, during the study period, an amount of USD 10,000 to USD 100,000 from Stryker Americas (Miramar, FL, USA).

All ICMJE Conflict of Interest Forms for authors and Clinical Orthopaedics and Related Research ${ }^{\mathbb{R}}$ editors and board members are on file with the publication and can be viewed on request. Each author certifies that his institution has approved the reporting of this case report and that all investigations were conducted in conformity with ethical principles of research.

G. L. Farfalli, P. A. I. Slullitel, D. L. Muscolo, M. A. Ayerza, L. A. Aponte-Tinao ( $\square)$

Carlos E. Ottolenghi Institute of Orthopedics, Italian Hospital of Buenos Aires, Potosí 4247 (1199), Buenos Aires, Argentina

e-mail: luis.aponte@hospitalitaliano.org.ar found 64 patients with a diagnosis of chondroblastoma of bone. After applying our selection criteria, 53 patients were involved in this study. We excluded seven patients with tumors initially treated with en bloc resection (five located in the extremities and two in the axial skeleton) and two patients with apophyseal tumors. One patient who underwent nonsurgical treatment and one patient lost to followup were also excluded. The mean age was 18 years (range, 11-39 years); the minimum followup was 2 years with a mean followup 77 months (range, 24-213 months). We analyzed all patients with a diagnosis of epiphyseal chondroblastoma of the limb treated with aggressive curettage and joint preservation surgery. During the period in question, our general indications for curettage were patients with active, painful tumors and those with more aggressive ones that remained intracompartmental, whereas initial wide en bloc resection was indicated in patients who had tumors with an extracompartmental extension breaching the adjacent joint cartilage and massive articular destruction. The tumor location was the distal femur in 14 patients, proximal tibia in 11, proximal humerus in 10, proximal femur in eight, the talus in seven, and elsewhere in the lower extremity in three. Local complications including joint degeneration and tumor recurrence were evaluated. Based on radiographic analysis, secondary osteoarthritis was classified by using the Kellgren-Lawrence grading system from Grade 0 to Grade IV. Patients who underwent joint replacement resulting from advanced symptomatic osteoarthritis were considered to have had joint failure for purposes of survivorship analysis, which was estimated using the Kaplan-Meier method. Functional results were evaluated with the Musculoskeletal Tumor Society functional score by the treating surgeon, who transcribed the results on the digital records every 6 months of followup. 
Results Twenty-two patients (42\%) developed 26 local complications. The most common local complication was osteoarthritis in 20 patients (77\% [20 of 26 complications]); tumor recurrence was observed in four patients; an intraarticular fracture and superficial infection treated with surgical débridement and antibiotics developed in one patient each. Joint survival was $90 \%$ at 5 years $(95 \%$ confidence interval [CI], 76\%-100\%) and $74 \%$ at 10 years (95\% CI, 48\%-100\%). Proximal femoral tumor location was associated with lower survivorship of the joint than other locations showing a 5-year survival rate of $44 \%$ (95\% CI, $0 \%-88 \% ; \mathrm{p}=0.000$ ). Of the 20 patients with osteoarthritis, four were symptomatic enough to undergo joint replacement, all of which were for tumors in the proximal femur. The mean Musculoskeletal Tumor Society functional score was 28 of 30 points (93\%).

Conclusions Osteoarthritis was a frequent complication of aggressive curettage of epiphyseal chondroblastoma, and tumors located in the proximal femur appeared to be at particular risk of secondary osteoarthritis and prosthetic replacement. Because chondroblastoma is a tumor that disproportionately affects younger patients, the patient and surgeon should be aware that arthroplasty at a young age is a potential outcome for treatment of proximal femoral chondroblastomas.

Level of Evidence Level III, therapeutic study. See Instructions for Authors for a complete description of levels of evidence.

\section{Introduction}

Chondroblastoma is an uncommon benign but locally aggressive tumor, most frequently located in the secondary centers of ossifications of long bones [9]. It represents $1 \%$ to $2 \%$ of all primary bone tumors and is more common in males than females (two to one), occurring most frequently in childhood and adolescence [20]. Clinical symptoms include pain aggravated by axial load; swelling; local tenderness; and diminished motion of the contiguous joint. It seldom presents as an incidental finding on plain radiographs [24]. The proximal humerus and the proximal femur are reported to be the most common locations [11, 20, 21].

The treatment of this tumor has traditionally been surgical excision. Aggressive curettage and bone grafting are usually performed to eradicate the tumor and maintain function, although local recurrences do occur. If bone grafting is not possible, alternatives include leaving the underlying bone cavity empty or packing it with polymethylmethacrylate [3]. More aggressive histological variants of chondroblastoma may be treated with en bloc resections with wide surgical margins, leading to larger residual osseous defects.
Aggressive curettage of epiphyseal tumors may result in damage to the growth plate and articular cartilage and over time may affect the functional outcome. To our knowledge, no studies have specifically addressed a comparison between radiographic and clinical outcomes with the risk of an arthroplasty or arthrodesis after initial curettage for the treatment of this tumor. Secondary osteoarthritis after aggressive curettage has been observed [3, 18, 20, 24]; nevertheless, in our review of the literature, no survival analyses have yet been reported.

We therefore asked: (1) What local complications can be expected after aggressive intralesional curettage of epiphyseal chondroblastoma? (2) What is the joint survival of a joint treated in this way for chondroblastoma? (3) What additional procedures are used in treating symptomatic joint osteoarthritis after treatment of the chondroblastoma? (4) What are the functional outcomes in this group of patients?

\section{Patients and Methods}

A retrospective review of our prospectively collected database between 1975 and 2013 was done, and all patients with a diagnosis of chondroblastoma of bone were analyzed. Every patient treated at our institution has a personal digital record entitled by the pathologic diagnosis and filled with demographic and clinical information as well as with scores regarding functional deficits that are completed by the corresponding treating surgeon. This study included only patients with a confirmed histological diagnosis, epiphyseal localization, and treated with intralesional resection at our institution with a minimum followup of 2 years. We found 64 patients with a diagnosis of chondroblastoma of bone. After applying our selection criteria, 53 patients were involved in this study. We excluded seven patients with tumors initially treated with en bloc resection (five located in the extremity and two in the axial skeleton) and two patients with apophyseal tumors. One patient who underwent nonsurgical treatment and one patient lost to followup were also excluded.

During the period in question, our general indications for curettage were patients with painful tumors and those more aggressive ones that remained intracompartmental, whereas initial wide en bloc resection was used for patients with tumors that had an extracompartmental extension breaching the adjacent joint cartilage with massive articular destruction.

Demographic characteristics as well as radiological, oncological, and clinical outcomes were reviewed by two investigators (GLF, PAIS), one of whom was not involved in the patients' original care (PAIS). The mean age at presentation was 18 years (range, 11-39 years) and mean 
followup was 78 months (range, 24-213 months). Thirtyseven were males. The most common localization was the distal femur affecting $26 \%$ of patients followed by the proximal tibia (Table 1). The most frequent nonlong bone site was the talus with $13 \%$. Thirty-seven tumors were considered Enneking [5] Stage 2 and 16 Stage 3. We detected no deaths nor metastases during followup.

Surgical approaches used were the standard for each joint involved. Adjuvant therapy included phenolization of the bone cavity after the curettage was done. In all patients, the standard treatment consisted of curettage and three cycles of phenolization of the bone cavity followed by application of $70 \%$ ethanol, always protecting the surrounding soft tissue, although there was some variation depending on the treating surgeon (DLM, MAA, LAA-T, GLF). After each cycle of phenolization, the cavity was extensively washed out by high-speed pulsatile lavage with saline solution. Regarding the proximal femoral epiphyseal tumors, a direct anterior or anterolateral approach was selected despite concerns about the vascular supply to the femoral epiphysis and the potential damage to the growth plate in the skeletally immature patients. In one patient, a medial approach was done (Ludloff-Ferguson approach [6, 16]) to remove a tumor located inferiorly and medially in the femoral head. In general the postoperative regimen was nonweightbearing for 2 to 3 months and then progressively increased to full weightbearing, but there was no standard protocol and this varied by surgeon and the patient.

We evaluated local complications in terms of recurrence and secondary osteoarthritis. Recurrence was detected by radiographs and CT of the operative site and confirmed by histological analysis. Patients were followed every 3 months during the first postoperative year, every 6 months during the second, and every year from then on. Radiographs of the operative site were obtained every 6 months during the first 2 years after curettage and every year from the third year on. Whenever symptoms or suspicious roentgenograms were encountered, further imaging studies such as CT scans and/or MRI were performed. Based on

Table 1. Location and number of chondroblastomas of bone in descending order of frequency

\begin{tabular}{ll}
\hline Location & Number of tumors \\
\hline Distal femur & 14 \\
Proximal tibia & 11 \\
Proximal humerus & 10 \\
Proximal femur & 8 \\
Talus & 7 \\
Distal tibia & 1 \\
Calcaneus & 1 \\
Metatarsal bone & 1 \\
\hline
\end{tabular}

radiographic analysis, we classified secondary osteoarthritis by using the Kellgren-Lawrence [10] grading system from Grade 0 to Grade IV. Joint failure for purposes of survivorship analysis was defined as a patient who underwent joint replacement or arthrodesis resulting from advanced symptomatic disease. Patients who exhibited signs of joint degeneration but remained asymptomatic were not considered joint failures. Other complications such as limb length discrepancies and growth arrests were also computed. They were only assessed in 18 skeletally immature patients with tumors located at the distal femur and proximal tibia nearby an open growth plate at the time of the surgery. All patients were clinically evaluated by the primary surgeon and a comparative full-leg radiograph was indicated if a notion of growth arrest was detected. Functional results were assessed with the Musculoskeletal Tumor Society (MSTS) score [5] by the treating surgeon, who transcribed the results on each patient's digital record every 6 months of followup.

Statistical analysis was performed using SPSS (Version 9.0; Chicago, IL, USA). The group comparison was made by means of confirmatory analysis. The Pearson chi square test was used to investigate nominally scaled data; significance levels were also determined. Joint cartilage survival over time was computed by Kaplan-Meier estimate. Survival analysis among different tumor locations was compared by log-rank test (Mantel-Cox).

\section{Results}

Twenty-two patients exhibited a local complication (42\%). Four recurrences (8\%) were observed at latest followup, all of which occurred in patients with Stage 3 tumors. They were all successfully treated by a second curettage and bone grafting (Table 2). The mean time to local recurrence was 11 months (range, 6-24 months). At a mean followup of 79 months (range, 35-180 months) after the second curettage, no new recurrences were diagnosed. The most frequent nononcological complication was secondary osteoarthritis, which developed in 20 patients (38\%).

We observed degenerative changes in six of 14 patients with distal femur tumors (three cases were Grade III and three grade IV); five of eight with proximal femur tumors (all of them were Grade IV); five of seven with talus tumors (one case was Grade III and four cases Grade IV); two of 10 with proximal humerus tumors (both cases were Grade III); and two of 11 with proximal tibia chondroblastomas after treatment (both Grade III). The mean time to appearance of osteoarthritis was 58 months (range, 27-240 months). Two other complications were found in patients with tumors located in the distal femur. An intraarticular posterior condyle fracture (Hoffa's fracture) occurred and 
Table 2. Oncological results

\begin{tabular}{lllllll}
\hline Site & $\begin{array}{l}\text { Enneking } \\
\text { stage }\end{array}$ & $\begin{array}{l}\text { Initial } \\
\text { treatment }\end{array}$ & $\begin{array}{l}\text { Time to recurrence } \\
\text { (months) }\end{array}$ & $\begin{array}{l}\text { Treatment } \\
\text { Metastatic } \\
\text { disease }\end{array}$ \\
\hline Distal femur & 3 & Curettage + BG & 6 & Curettage + BG & 35 & $\begin{array}{l}\text { Time from recurettage } \\
\text { to latest followup (months) }\end{array}$ \\
Proximal femur & 3 & Curettage + BG & 24 & Curettage + BG & 62 & - \\
Metatarsal bone & 3 & Curettage + BG & 6 & Curettage + BG & 180 & - \\
Proximal tibia & 3 & Curettage + BG & 7 & Curettage + BG & 39 & - \\
\hline
\end{tabular}

$\mathrm{BG}=$ bone grafting.

was treated by open reduction, internal fixation, and new bone grafting. Another patient developed a superficial infection treated by surgical débridement and antibiotics. No growth arrests nor limb length discrepancies were detected in the 18 skeletally immature patients with chondroblastomas of the distal femur or proximal tibia.

Joint cartilage survival was $90 \%$ at 5 years postoperatively $(95 \%$ confidence interval $[\mathrm{CI}], 76 \%-100 \%)$ and $74 \%$ at 10 years $(95 \%$ CI, $48 \%-100 \%)$ (Fig. 1). The proximal femur showed the poorest joint cartilage survival compared with other locations showing a 5-year survival of $44 \%$ (95\% CI, $0 \%-88 \% ; \mathrm{p}=0.000$ ).

Four of the 20 patients with secondary osteoarthritis, all of whom had proximal femoral tumors (Fig. 2), were sufficiently symptomatic to undergo total joint arthroplasty. The average time from initial treatment to THA was 120 months (range, 96-180 months). The remaining patients who showed evidence of secondary osteoarthritis were treated without surgery as a result of asymptomatic or minimally symptomatic disease.

The mean MSTS score was 28 of 30 points (93\%) (range, 21-30; SE 2.6), which is considered to be an excellent score. Most of the patients had no functional deficits. With the numbers we had, we could not demonstrate a lower score for patients with tumors located at the proximal femur (24 of 30 [80\%]; range, 21-30; SE 3.5; Table 3).

\section{Discussion}

Aggressive curettage remains the mainstay of treatment for chondroblastoma of bone, because it is associated with a low likelihood of metastases and local recurrences [24, 26]. However, considering that this epiphyseal tumor affects primarily children and young adults, the question arises whether this treatment approach also results in durable preservation of the patient's native articular surface over the years after the initial treatment. We therefore studied a group of patients with epiphyseal chondroblastoma of

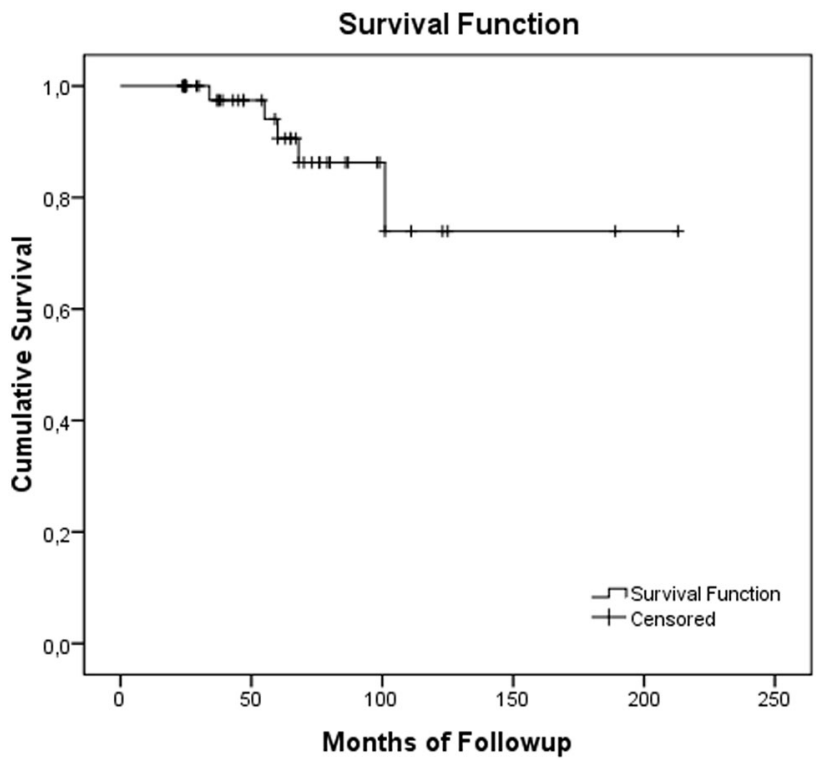

Fig. 1 Graphic showing the overall joint cartilage survival obtained by the Kaplan-Meier estimate.

bone, paying particular attention to local complications and survivorship of the joint itself.

Our study had certain limitations. First, there is the issue of heterogeneity. This series included tumors in various anatomic locations, and some sites had relatively few tumors for analysis. Moreover, many factors may have been associated with the genesis of osteoarthritis. In addition to the effect an epiphyseal location of a lesion might have on the articular cartilage, iatrogenic joint damage from excessive curettage, disruption of the bone's main vascular supply after a direct approach, and chemical injury secondary to phenolization could all be related individually or collectively to the onset of secondary osteoarthritis. Also, growth arrest is of more importance for tumors about the knee than at other sites and obviously would occur only in patients who were skeletally immature; because we had few such patients, we cannot accurately assess the frequency of this event. These issues 


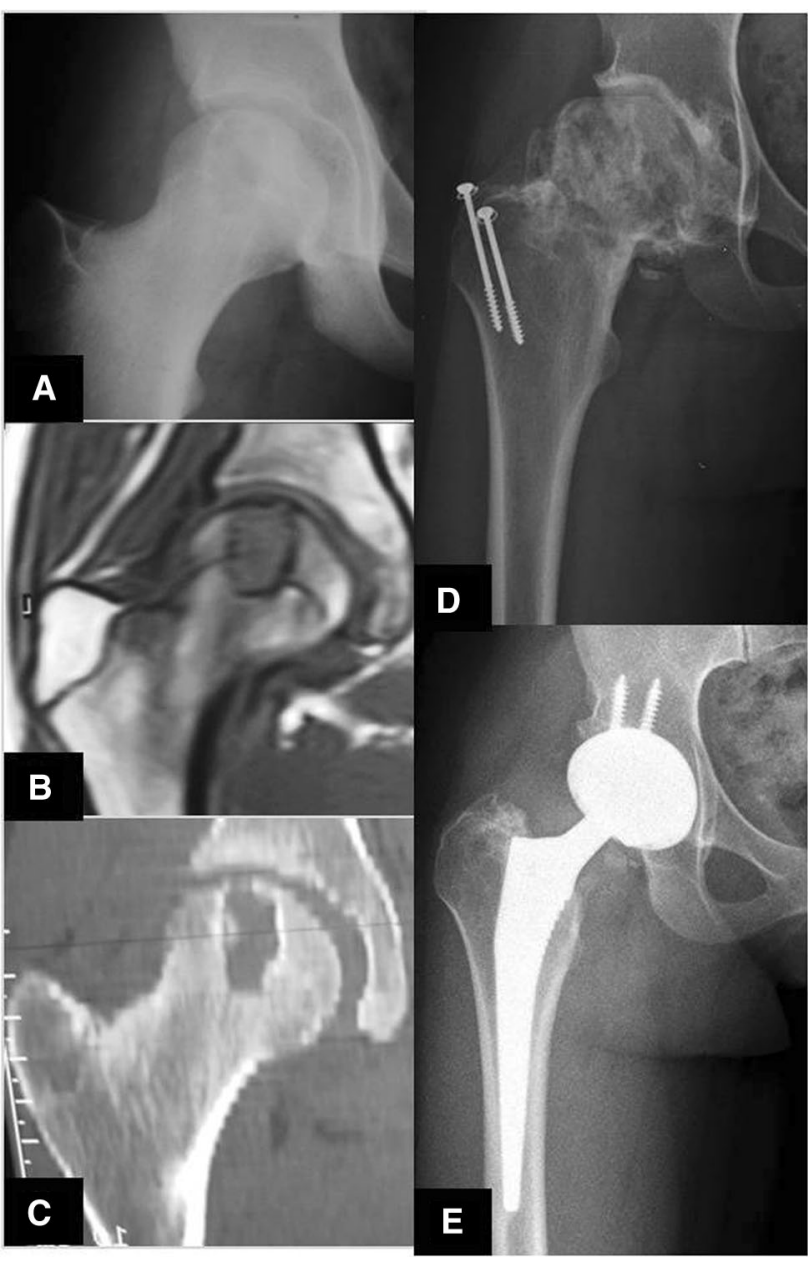

Fig. 2A-E (A-D) A 16-year-old boy with a chondroblastoma affecting the epiphysis of the right hip. (A) AP radiograph of the right hip at the moment of diagnosis shows the lesion in the epiphysis of the proximal femur; (B) coronal MR image of the proximal femur shows the tumor in contact with the articular cartilage; (C) CT of the proximal right femur shows the compromise of the epiphysis and the sclerotic rim around the lesion. (D) AP radiograph of the right hip shows signs of severe joint degeneration 6 years after the initial curettage. (E) AP radiograph of the right hip shows an uncemented THA.

should be taken into consideration when interpreting our survival estimates. Second, we did not consider asymptomatic patients who exhibited signs of joint degeneration as joint failures for purposes of survivorship analysis. Because these patients are young, we expect that many of these patients may yet undergo arthroplasty at longer followup; that is, our survivorship figures should be considered best-case estimates. Third, given the exclusion of axial and aggressive tumors, our results of oncological outcome may also represent a best-case estimate of recurrence for patients with chondroblastomas overall. The incidence of metastasis secondary to chondroblastoma is not well defined in our study, but it is believed to occur rarely, in fewer than $1 \%$ of patients $[12,22]$. It is also possible that we did not detect possible metastases given our protocol of imaging post-treatment, which did not include routine chest CT scans at periodic intervals. Fourth, we believe the MSTS score is not an ideal one to use for assessment of functional results after curettage of benign epiphyseal tumors of the limb. Although functional evaluation with this score was made prospectively, it might be considered permissive and not joint-specific for these tumors in which more aggressive limb salvage procedures are performed. As a consequence, no statistical difference was found between tumors among different locations, despite the observation that patients with hip tumors proved to be more symptomatic.

A total of $42 \%$ of our patients experienced local complications (22 of 53 patients), of which the most frequent one was secondary osteoarthritis seen in $38 \%$ of cases $(20$ of 53 patients). It seems likely that the high risk of degenerative arthritis was related to the initial aggressiveness of the treatment. Injury to the cartilage and avascular necrosis are potential risks that should be foreseen when considering a direct approach for tumor excision. Orthopaedic sequelae like these have been mentioned by others $[18,20,22,24]$; however, to our knowledge, this is the first report evaluating survival analysis of the joint. We found that with our approach of aggressive curettage, local recurrence was uncommon, but secondary arthritis was frequent. This is perhaps not surprising; we see this as a tradeoff, but the first responsibility of the oncologic surgeon is to minimize the likelihood of local recurrence, which represents a more severe complication than does secondary arthritis. We therefore believe that the more

Table 3. Mean MSTS functional score according to tumor location

\begin{tabular}{|c|c|c|c|c|c|c|c|}
\hline Tumor location & Pain & Function & Emotional acceptance & Supports & Walking & Gait & Total \\
\hline Proximal femur & 3 & 4 & 5 & 4 & 4 & 4 & 24 \\
\hline Distal femur & 4 & 5 & 5 & 5 & 5 & 5 & 29 \\
\hline Proximal tibia & 4 & 5 & 5 & 5 & 5 & 5 & 29 \\
\hline Proximal humerus & 5 & 4 & 5 & 5 & 5 & 5 & 29 \\
\hline Foot and ankle & 4 & 5 & 5 & 5 & 5 & 4 & 28 \\
\hline
\end{tabular}

Foot and ankle location included tumors sited at the distal tibia, talus, calcaneus, and metatarsal bone; MSTS = Musculoskeletal Tumor Society. 
aggressive the curettage is, the worse joint survival results and the lower the recurrence remains. Approximately $8 \%$ (four of 53) experienced recurrences. Sailhan et al. [20] reported that $32 \%$ developed recurrences, analyzing only pediatric patients. They concluded that a less extensive curettage might have been performed in young patients as a result of concerns about the adjacent open physis. Suneja et al. [24] observed local recurrences in $13 \%$ of patients, claiming that the underlying cause was incomplete curettage because surgeons were understandably concerned about damaging the growth plate. We consider the growth plate to be resilient [4] and, with proper consideration (most patients are near the completion of longitudinal growth or have tumors at sites of less importance with respect to growth), aggressive curettage should be performed even in the proximity of the physis. We did not observe any clinically relevant growth arrest, limb length discrepancies, or malalignments as complications. Many authors have reported that tumors in the proximal femur and the pelvis are more likely to recur as a result of the difficulty in the surgical access necessary to achieve complete excision $[15,18,22,24]$. We have described only one proximal femur recurrence after performing a direct anterior or anterolateral approach in most cases. As previously stated by Strong et al. [23], chondroblastomas of the femoral capital epiphysis are surrounded by articular cartilage on one side and by physeal cartilage on the other. Therefore, it may be challenging to decide between a minimally invasive approach by drilling the lateral femoral cortex up through the neck or an open approach raising a window through the femoral head or neck. Like Strong et al. [23], we believe a direct approach should be performed whenever possible because it allows visualization of the entire lesion, although vascular, articular, or even physeal injury remain as possible complications.

Compared with other sites, more frequent progression to secondary osteoarthritis appeared to be more common in patients with hip and talus chondroblastomas, although we did not confirm this with a statistical analysis because of small numbers of patients at these sites. Degenerative changes in these joints might have been related to their unique vascular supply. The intracapsular portion of the femoral neck has essentially no cambium layer in its fibrous covering to participate in peripheral callus formation, making healing dependent mostly on the endosteal, not periosteal, surface [8]. Hence, femoral head nutrition is reliant on preserving the integrity of the retinacular vessels, which can be disrupted during the surgical approach. Similarly, the talus has two features that relate to its nutrition: a high percentage of surface covered by articular cartilage and a retrograde blood supply [17]. Like displaced fractures of the talar neck, a direct approach through a bone window may disrupt the arteries to the tarsal canal and to the tarsal sinus, which are the main vascular supply. We used phenolization as adjuvant therapy that could cause damage to the articular cartilage. Although Lehner et al. [14] found inconclusive evidence to support the use of adjuvant therapy, additional chemical (phenol, liquid nitrogen) or thermal adjuvants (radiofrequency, electrocautery, argon bean coagulation, cryotherapy) can aid in preventing recurrence when used cautiously $[9,18,20]$. Phenolization has been associated with a lower risk of osteoarthritis than liquid nitrogen when treating similar tumors such as giant cell tumors [1, 25].

Joint cartilage survival was poorest in patients with chondroblastomas in the proximal femur than other locations. Severe joint space narrowing at the hip in active, young, and otherwise healthy patients led to the performance of total joint arthroplasty in four patients with proximal femur lesions. Historically, there has been no direct association between radiological findings and the indication for THA. Gossec et al. could not find a cutpoint for pain and/or physical disability that accurately discriminated patients who did versus who did not receive a total joint arthroplasty recommendation by their physicians [7]. However, they found radiographic severity and symptom levels as factors that predicted total joint arthroplasty. Because arthritis occurred in more than half of our patients with proximal femur lesions, one might consider alternative approaches such as radiofrequency ablation and grafting through a more limited approach [13, 19]. Despite careful surgical dislocation with attention to preserving the epiphyseal vessels, the femoral head sphericity was not retained in many of our patients and that incongruence of the ball and socket resulted in symptomatic hips needing a second surgical procedure [2].

With the numbers we had, we could not show that our functional outcome with hip tumors was different than that at other sites. Because chondroblastoma is a benign bone tumor and is treated with a rather simple procedure, mean MSTS score of the overall series was excellent with most of the patients having no functional deficits and was probably not sufficiently selective to show difference which might exist.

In conclusion, our findings suggest that aggressive curettage and bone grafting were successful in treating the tumor in all but four of our 53 patients. Nevertheless, osteoarthritis was a frequent complication of this procedure in these sites, which are adjacent to a joint. In fact, arthritis and local recurrence may be even more common than we estimated it here, because these patients are young, and both of those complications may become more frequent over time. Furthermore, secondary osteoarthritis of the proximal femur seems to be more symptomatic than in other locations and may be the reason why four of our eight patients elected to have a prosthetic hip replacement. 
Because chondroblastoma occurs in young patients, and our results likely underrepresent the eventual joint sequelae, secondary arthritis should be considered when planning treatment at this site and patients should be aware of this potential outcome.

\section{References}

1. Becker WT, Dohle J, Bernd L, Braun A, Cserhati M, Enderle A, Hovy L, Matejovsky Z, Szendroi M, Trieb K, Tunn P-U. Local recurrence of giant cell tumor of bone after intralesional treatment with and without adjuvant therapy. J Bone Joint Surg Am. 2008;90:1060-1067.

2. Bowman KF, Fox J, Sekiya JK. A clinically relevant review of hip biomechanics. Arthroscopy. 2010;26:1118-1129.

3. Campanacci M, Bertoni F, Bacchini P. Bone and Soft Tissue Tumors: Clinical Features, Imaging, Pathology, and Treatment. $2^{\text {nd }}$ ed. Vienna, Austria: Springer-Verlag; 1999.

4. Cool WP, Carter SR, Grimer RJ, Tillman RM, Walker PS. Growth after extendible endoprosthetic replacement of the distal femur. J Bone Joint Surg Br. 1997;79:938-942.

5. Enneking WF, Dunham W, Gebhardt MC, Malaward M, Pritchard DJ. A system for the functional evaluation of reconstructive procedures after surgical treatment of tumors of the musculoskeletal system. Clin Orthop Relat Res. 1993;286:241-246.

6. Ferguson AB Jr. Primary open reduction of congenital dislocation of the hip using a median adductor approach. J Bone Joint Surg Am. 1973;55:671-689.

7. Gossec L, Paternotte S, Maillefert J, Cambescure C, Conaghan P, Davis A, Gunther K. The role of pain and functional impairment in the decision to recommend total joint replacement in hip and knee osteoarthritis: an international cross-sectional study of 1909 patients. Report of the OARSI-OMERACT Task Force on total joint replacement. Osteoarthritis Cartilage. 2011;29:997-1003.

8. Grose AW, Gardner MJ, Sussmann PS, Helfet DL, Lorich DG. The surgical anatomy of the blood supply to the femoral head: description of the anastomosis between the medial femoral circumflex and inferior gluteal arteries at the hip. J Bone Joint Surg Br. 2008;90:1298-1303.

9. Jaffe H, Lichteinstein L. Benign chondroblastoma of bone. A reinterpretation of the so-called calcifying or chondromatous giant cell tumor. Am J Pathol. 1942;XVIII:969-991.

10. Kellgren JH, Lawrence JS. Radiological assessment of osteoarthrosis. Ann Rheum Dis. 1957;16:494-502.
11. Kolodny A. Bone sarcoma: the primary malignant bone tumors of bone and the giant-cell tumour. Surg Gynecol Obstet. 1927;44(Suppl):178-183.

12. Kyriakos M, Land VJ, Penning HL, Parker SG. Metastatic chondroblastoma. Report of a fatal case with a review of the literature on atypical, aggressive, and malignant chondroblastoma. Cancer. 1985;55:1770-1789.

13. Lalam RK, Cribb GL, Tins BJ, Cool WP, Singh J, Tyrrell PN. Image guided radiofrequency thermo-ablation therapy of chondroblastomas: should it replace surgery? Skeletal Radiol. 2014;43:513-522.

14. Lehner B, Witte D, Weiss S. Clinical and radiological long-term results after operative treatment of chondroblastoma. Arch Orthop Trauma Surg. 2011;131:45-52.

15. Lin PP, Thenappan A, Deavers MT, Lewis VO, Yasko AW. Treatment and prognosis of chondroblastoma. Clin Orthop Relat Res. 2005;438:103-109.

16. Ludloff $\mathrm{K}$. The open reduction of the congenital hip dislocation by an anterior incision. Am J Orthop Surg. 1913;10:438-454.

17. Mulfinger GL, Trueta J. The blood supply of the talus. J Bone Joint Surg Br. 1970;52:160-167.

18. Ramappa A, Lee F, Tang P, Carlson J, Gebhardt M, Mankin H. Chondroblastoma of bone. J Bone Joint Surg Am. 2000;90:571-578.

19. Rybak LD, Rosenthal DI, Wittig JC. Chondroblastoma: radiofrequency ablation-alternative to surgical resection in selected cases. Radiology. 2009;251:599-604.

20. Sailhan F, Chotel F, Parot R. Chondroblastoma of bone in a pediatric population. J Bone Joint Surg Am. 2009;91:2159-2168.

21. Schajowicz F, Gallardo H. Epiphysial chondroblastoma of bone: a clinico-pathological study of sixty-nine cases. J Bone Joint Surg Br. 1970;52:205-226.

22. Springfield D, Capanna R, Campanacci M. Chondroblastoma. A review of seventy cases. J Bone Joint Surg Am. 1985;67:748-755.

23. Strong DP, Grimer RJ, Carter SR, Tillman RM, Abudu A. Chondroblastoma of the femoral head: management and outcome. Int Orthop. 2010;34:413-417.

24. Suneja R, Grimer RJ, Belthur M, Jeys L, Carter SR, Tillman RM, Davies AM. Chondroblastoma of bone: long-term results and functional outcome after intralesional curettage. J Bone Joint Surg Br. 2005;87:974-978.

25. van der Heijden L, van der Geest IC, Schreuder HW, Dijkstra PD. Liquid nitrogen or phenolization for giant cell tumor of bone? $J$ Bone Joint Surg Am. 2014;35:1-9.

26. Xu H, Nugent D, Monforte HL, Binitie OT, Ding Y, Letson GD, Cheong D, Niu X. Chondroblastoma of bone in the extremities: a multicenter retrospective study. $J$ Bone Joint Surg Am. 2015;97:925-931. 